\title{
ALTIMETER AS AN IMAGER OF THE SEA SURFACE ROUGHNESS: COMPARISON OF SAR AND LRM MODES
}

\author{
Jean Tournadre, Bertrand Chaprono \\ Laboratoire d'Océanographie Physique et Spatiale, \\ IFREMER, Univ. Bretagne Occidentale, CNRS, IRD, \\ Plouzané, 29280, France
}

\begin{abstract}
Satellite altimeters, designed to measure the topography of the ocean, are also good imagers of the ocean surface backscatter. A method was developed to invert the classical Low Resolution Mode high resolution HR waveforms in terms of surface backscatter at high resolution $(\sim 300 \mathrm{~m})$. Since the launch of Cryosat-2 in 2010, a new generation of altimeters using Doppler capabilities has emerged and has become the standard for the new altimeters such as the Sentinel-3 one. The so-called SAR altimetry provides higher along-track resolution and lower noise level. In this mode altimeter are also good imagers of the surface backscatter. A new method of inversion of SAR waveforms is presented. The processing of the Sentinel-3 altimeters provides collocated SAR and LRM waveforms that are used to intercompare the inverted surfce backscatter.
\end{abstract}

Index Terms - Altimeter, high resolution surface roughness, inversion

\section{INTRODUCTION}

Conventional satellite altimeters used pulse-limited-geometry and full deramp technique to measure the power backscatter by the ocean surface as a function of time, i.e. the altimeter pulse echo waveforms (WF). Over an ocean surface, the WF has a characteristic shape that can be described analytically using in general the classical Brown model [1]. The altimeter geophysical parameters: epoch (range), surface backscatter, and significant wave height as well as the satellite off-nadir angle are estimated by fitting the theoretical model to the measured WFs. The basic assumption of the echo WFs models is that the distribution of sea surface roughness within the altimeter footprint is homogeneous and can be described with a mean value. However, as clearly shown by numerous SAR images of the sea surface

this basic hypothesis is not always valid notably in the presence of rain, sea ice, small islands or reefs, surface slicks or very low wind conditions.

Thanks to Centre National d'Etudes Spatiales for funding.
Under such occurrences, an altimeter can be seen as an imager of the sea surface backscatter whose imaging process is more complex than a classical one in the sense that pixels are not rectangular but annular. The imaging process of the sea surface, i.e. the transform matrix between the real and the WF spaces, depends only on the satellite and altimeter geometry and can be analytically computed. A method to invert the measured WFs in terms of surface backscatter at high resolution (HR) ( $300 \mathrm{~m})$ was developed by [2].

Since the launch of Cryosat-2 in 2010, a new generation of altimeters using Doppler capabilities has emerged and has become the standard for the new altimeters such as the Sentinel3 ones [3]. The Delay-Doppler Altimeter (DDA) concept (or SAR altimetry), first proposed by [4]. The use of the surface Doppler information allows to increase the along track a resolution while the combination (or "stacking") of different samplings of the same Doppler strip under different incidences allows to strongly reduce the speckle noise. In SAR mode, altimeter can also be considered as imager of the sea surface roughness and a method of inversion of SAR echo WFs in terms of HR surface backscatter has been developed.

The Sentinel-3 processing produces both SAR and PseudoLRM (similar to conventional altimeter) echo WFs and provides thus a good base for the inter-comparison of SAR and LRM inverted backscatter.

\section{ALTIMETER WF INVERSION IN TERMS OF SURFACE BACKSCATTER}

\subsection{Inversion of Low Resolution Model (LRM) WFs}

In LRM mode, altimeters operate in the classical pulse limited mode. The echo WF can be represented in analytical form by the classical Brown model [1]

$$
\sigma(t)=\frac{1}{2}(2 \pi)^{3 / 2} H^{\prime \prime} \sigma_{\tau} \sigma_{0}\left(1+\operatorname{erf}\left(\frac{x}{\sqrt{2} \sigma_{p}}\right)\right) e^{-\frac{x}{u_{b}}}
$$

where $t$ is the time, $x=c t / 2$ the distance between the surface and the antenna, $u=\left(H^{\prime} \psi^{2}\right) / 2$ the range, $H^{\prime}$ and $H^{\prime \prime}$, de- 


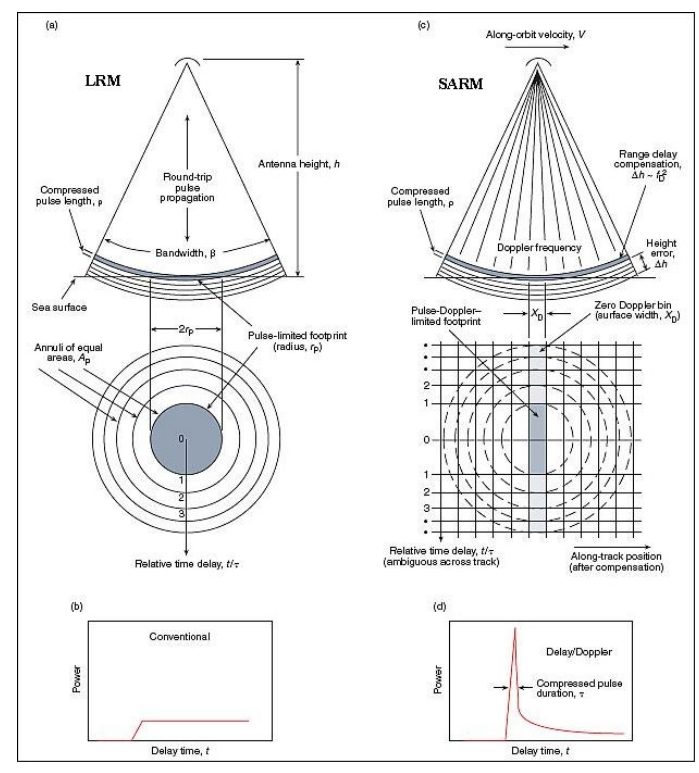

Fig. 1. Comparison of a conventional pulse-limited radar altimeter's (a) illumination geometry (side view) and footprint (plan view) and (b) impulse response, with a delay/Doppler altimeter's (c) illumination geometry and footprint and (d) impulse response (Credits R.K. Raney, Johns Hopkins University Applied Physics Laboratory)

fined by $H^{\prime}=H(1+H / a)$ and $H^{\prime \prime}=H /(1+H / a)$, are the reduced and extended satellite heights, $H$ is the satellite altitude and $a$ the earth's radius; $R(0)$ is the Fresnel coefficient at 0 incidence, $u_{b}$ is defined by $u_{b}=\left(H^{\prime} \psi_{b}^{2}\right) / 2$, with $\psi_{b}=\psi_{H} / \sqrt{8 \ln 2}, \psi_{H}$ is the two-way half-power antenna beam-width. $\sigma_{p}$ is defined by $\sigma_{p}=\sqrt{h^{2}+\sigma_{\tau}^{2}}$; $h$ is the rms wave height and $\sigma_{0}$ is the mean surface backscatter coefficient. Note that $t=0$ corresponds to the mean sea level. The main assumption of the Brown model is that the sea surface within the altimeter footprint can be represented by a mean surface backscatter $\sigma_{0}$.

The method of inversion has been described in detail by[2] and is only outlined here. High resolution (HR) altimeter WFs are given every $0.05 \mathrm{~s}$ in telemetry bins of width $\tau$ (length of the pulse, 3.125ns). The distance between two consecutive HR WFs is of the order of $300 \mathrm{~m}$. The number of bins is in general 128 and the nominal track point (corresponding to the mean sea level or $t=0$ ) is shifted around 40 . For a flat sea, the telemetry bins range varies from $\sim 1 \mathrm{~km}$ at bin 1 to $\sim 8 \mathrm{~km}$ at bin 104 . The distance between two consecutive bins, i.e. the width of the annular pixel, varies from about $2 \mathrm{~km}$ at nadir (circular) to $60 \mathrm{~m}$ at bin 104 . The section of the WFs above the sea level are discarded in the analysis.

Assuming that the surface backscatter $(\sigma)$ is defined on a regular along-track grid $\left\{x_{k l}, y_{k l}\right\}$, and assuming that the rms of the surface is small (low significant wave height), the Brown echo WF [1] can be expressed in a discrete form as

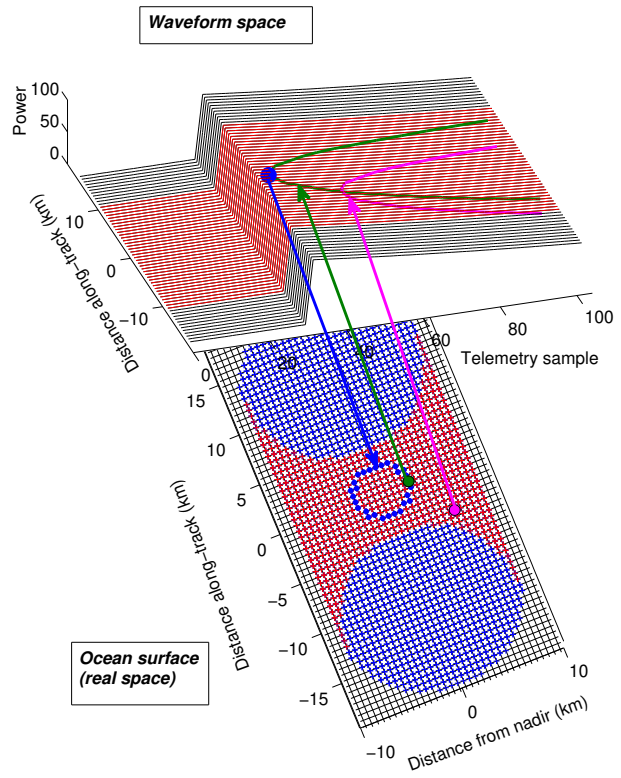

Fig. 2. Altimeter imaging process. Lower plot: sea surface regular grid (real space RS). Upper plot: WF space (WS). The green (magenta) parabola represents the ensemble of the WS bins to which the green (magenta) surface point contributes. The blue annulus in the RS represents the ensemble of surface elements that contribute to the blue bin of the WF space. The group of red WFs is the image of the ensemble of surface blue points. The red surface points are the one that contribute only to the purple WFs.

follow [2], the $j^{\text {th }}$ element of the $i^{t h}$ WF $w_{i j}$ is the summation of the surface backscatter elements $\sigma_{k l}$ whose range is between the range limits $\left\{u_{j}, u_{j+1}\right\}$ of bin $j$,

$$
w_{i j}=\alpha \sum_{k} \sum_{l} a_{k l} \sigma_{k l} e^{-\frac{u(k, l)}{u_{b}}}\left(1+\operatorname{erf}\left(\frac{u_{j}}{\sqrt{2} \sigma_{p}}\right)\right)
$$

where the range $u_{k l}$ satisfies $u_{j} \leq \frac{\left(x_{k l}-x_{i}^{0}\right)^{2}+\left(y_{k l}-y_{i}^{0}\right)^{2}}{H^{\prime \prime} c \tau} \leq$ $u_{j+1}$ and where $\tau$, pulse length, $\{x, y\}$ along and across track coordinates, $\left\{x_{i}^{0}, y_{i}^{0}\right\}$ the coordinates at nadir of the $i^{t h} \mathrm{WF}$ and $a_{k l}$ surface of the intersection of the $\{k, l\}$ grid cell and the annulus of bin $j$. In a first order approximation we assume all $u_{k l}$ to be equal to $\overline{u_{j}}=\left(u_{j}+u_{j+1}\right) / 2$, thus Eq. (2) simplifies to

$$
\mathbf{W}_{i j}=w_{i j} \frac{e^{\frac{\overline{u_{j}}}{u_{b}}}}{\left(1+\operatorname{erf}\left(\frac{\overline{u_{j}}}{\sqrt{2} \sigma_{p}}\right)\right)}=\alpha \sum_{k, l} a_{k l} \sigma_{k l}
$$

where $\mathrm{W}$ is the WF detrended for the beam-width by the term $e^{\frac{\overline{u_{j}}}{u_{b}}}$ and wave height effects by the term $\left(1+\operatorname{erf}\left(\frac{\overline{u_{j}}}{\sqrt{2} \sigma_{p}}\right)\right)^{-1}$. 
The inversion of a single WF in term of surface $\sigma$ is obviously impossible but if we consider a series of detrended WF $\left\{\mathrm{W}_{\mathrm{i}}, i=1 . . N\right\}$ (in red in Figure 2), they can be expressed in matrix form as

$$
\mathrm{W}=\mathrm{A} . \mathrm{S}
$$

where $S$ is the matrix of the mean left/right surface backscatter (because of the left/right ambiguity) and $A$ is the altimeter imaging matrix that can be easily computed using the range.

Let $X_{i j}$ be a surface grid element of area $d x, d y$ centered on $x_{i j}, y_{i j}$. The coefficient $a_{i j k l}$ of $\mathrm{A}$ is equal to the surface of intersection between the grid element and the annulus centered at $x_{0}^{k}, y_{0}^{k}$ and radii $r_{l}$ and $r_{l+1}$ (i.e. bin $l$ range).

$$
a_{i j k l}=\int_{y_{i j}-\frac{d y}{2}}^{y_{i j}+\frac{d y}{2}}\left[f_{1}(y)-f_{2}(y)\right] d y
$$

where $f_{1}(y)=\min \left(\sqrt{r_{l+1}^{2}-\left(y-y_{0}^{k}\right)^{2}}, x_{i j}+\frac{d x}{2}\right)$, $f_{2}(y)=\max \left(\sqrt{r_{l}^{2}-\left(y-y_{0}^{k}\right)^{2}}, x_{i j}-\frac{d x}{2}\right)$ and $r_{l}=\sqrt{l H^{\prime \prime} c \tau}$. The $\sigma$ grid resolution is chosen as the distance between two consecutive HR WFs (290 m for Jason). The minimum number of WFs to be considered is constrained by the width of the image of a nadir point in the WF space that is about $3 \mathrm{sec}-$ onds of data or 60 WFs. In practice, $\mathrm{N}$ has been fixed to 75 . For such grids, the linear system of Equations (4) is overdetermined and can be inverted using pseudo Moore-Penrose inverse $\mathrm{A}^{+}$computed using singular value decomposition [5].

$$
\mathrm{S}=\mathrm{A}^{+} \cdot \mathrm{W}
$$

For each $20 \mathrm{~Hz} \mathrm{WF}$, the 75 surrounding WFs are considered and the local $\mathbf{S}$ matrix is estimated using Eq (6). Only the subset of points whose images are completely included in the WFs are kept and the local matrices are then averaged to produce the final estimate of the along track $\sigma$.

A main assumption of the inversion method is that the rms of the sea surface elevation is low, which is quite a strong constraint. However, higher sea state $(>2 \mathrm{~m})$ are most probably associated, except for swell events, to medium to high winds that wipe away the small scale variations of surface backscatter. In such cases, high resolution mapping of surface backscatter is certainly of little interest.

\subsection{Inversion of Delay Doppler (SAR) altimeter WF}

The Delay Doppler or SAR altimeter mode (first proposed by [4]) was successfully tested on board Cryosat and it is now the standard operating mode of Sentinel 3 altimeters. In this mode, the altimeter transmits bursts with a frequency of about $85 \mathrm{~Hz}$ [3]. Each burst contains 64 coherent pulses (transmitted at a $18,182 \mathrm{~Hz}$ PRF) which are measured over time windows of 128 bin length $(60 \mathrm{~m})$ and are then processed by along-track FFT to obtain the Delay Doppler map (DDM) of the surface-reflected signal. Sixty-four Doppler beams of equal angular sectors are thus obtained. Each Doppler beam is about $250 \mathrm{~m}$ wide in the along-track direction and the interval between bursts corresponds to the satellite moving forward by $80 \mathrm{~m}$ each time in SAR mode. The strips laid down by successive bursts can be "stacked" to obtain multiple looks of the same portion of the surface. After range alignment including slant, tracker and Doppler range corrections and after range compression [6, 7], stacks of co-located Doppler beams (L1B-S data) are produced. The incoherent summation of the L1b-S finally gives the SAR Echo (or WF). An analytical model of SAR echo was proposed by [6].

The SAR WF in discrete form in the same way as the LRM one, the $j^{\text {th }}$ element of the $i^{\text {th }} \mathrm{WF} w_{i j}$ is the summation of the surface backscatter elements $\sigma_{k l}$ whose range is between the range limits $\left\{u_{j}, u_{j+1}\right\}$ of bin $j$ and whose along-track coordinates is between $\left\{y_{i}^{0}-d y / 2, y_{i}^{0}+d y / 2\right\}$ where $y_{i}^{0}$ is the along track nadir coordinate of the WF and $d y$ the along-track distance between two WFs.

$$
w_{i j}=\alpha \sum_{k} \sum_{l} a_{k l} \sigma_{k l} e^{-\frac{u(k, l)}{u_{b}}}\left(1+\operatorname{erf}\left(\frac{u_{j}}{\sqrt{2} \sigma_{p}}\right)\right)
$$

The use of the Doppler information simplifies the computation of the $a_{k l}$ coefficients as it depends only on the range distance

$$
a_{k l}=a_{k}=2\left(r_{k}-r_{k+1}\right) d y
$$

where $r_{k}=\sqrt{\frac{H}{1+H / a} k c \tau}$ is the range of the bin $k$.

$$
\mathrm{W}_{i j}=w_{i j} \frac{e^{\frac{\bar{u}}{u_{b}}}}{\left(1+\operatorname{erf}\left(\frac{\overline{u_{j}}}{\sqrt{2} \sigma_{p}}\right)\right)}=\alpha a_{j} \sigma_{i, j}
$$

It is thus possible to invert a single WF in term of across-track $\sigma$ on a grid of variable resolution $\left\{r_{k}, k=1: m\right\}$.

$$
\sigma_{i j}=W_{i j} /\left(\alpha a_{j}\right)
$$

Figures 3 and 4 present an exemple of inversion of SAR and LRM WF over a large pumice raft that resulted from an eruption of a submarine volcano near the Date island of Tonga in august 2019. On August 23 the raft was located near $176^{\circ} \mathrm{W}, 18.5^{\circ} \mathrm{S}$ and was sampled by Sentinel3-B. The raft is visible the OLCI image under the semi-transparent clouds. The Ku and $\mathrm{C}$ bands PLRM and KU band SAR WF have been inverted in terms of HR surface $\sigma$. The resulting fields presenting in figure 4 clearly shows the signature of the pumice that kills the short surface capillary waves. Although the noise level is significantly higher for PRLM WF it is remarkable to note that the PLRM inverted $\sigma$ is very consistent with the SAR one and that its noise level is comparable. This results from the fact that the use of 75 waveforms and the averaging process in the LRM inversion is similar to the use of the Doppler information and stacking. 


\section{PERSPECTIVES}

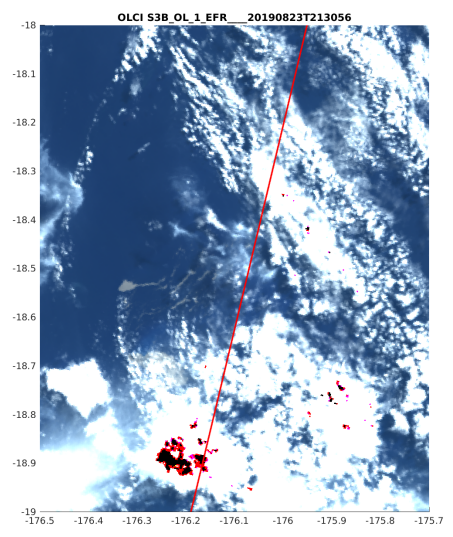

Fig. 3. Sentinel3B OLCI image on Aug. 23, 2019 21:30 UT over a large pumice raft near Tonga in the Pacific
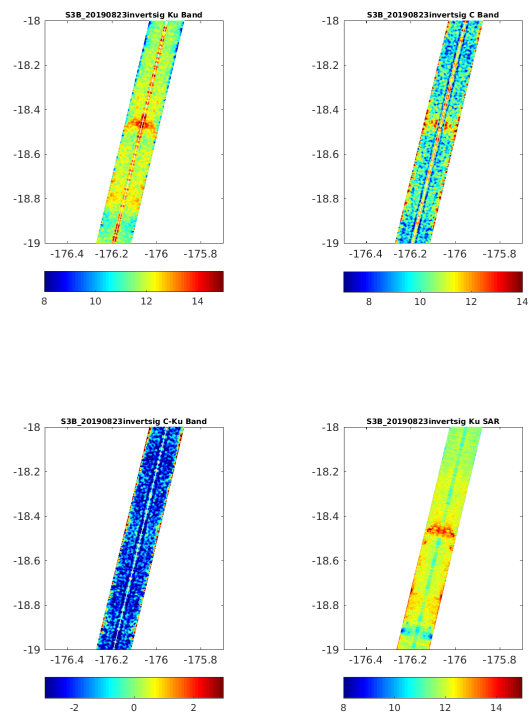

Fig. 4. Inverted PLRM Ku band $\sigma$ (top left), PRLM C band $\sigma$ (top right), C-Ku band $\sigma$ (bottom left) and inverted SAR $\sigma$ (bottom right). Sentinel3-B on Aug. 23, 2019 21:30 UT.
Both altimeter SAR and LRM modes WF can be inverted in terms of high resolution surface backscatter. The LRM and SAR inversions are of comparable quality, mainly because the LRM inversion capitalizes on the inversion and averaging over multiple waveforms in a certain way similar to the stacking process. This shows that the processing of altimeters LRM archives can/could provide HR surface $\sigma$ with a noise level comparable to SAR altimeter data. Such data can be use to study surface film (or oil spill) [8] or internal wave signatures.

\section{REFERENCES}

[1] G. S. Brown, "The average impulse response of a rough surface and its applications," IEEE Trans. Antennas Propag.brekke, vol. AP-25, pp. 67-74, 1977.

[2] J. Tournadre, B. Chapron, and N Reul, "High-resolution imaging of the ocean surface backscatter by inversion of altimeter waveforms," J. Atmos. Oceanic Technol., vol. 28, pp. 1050-1062, 2011.

[3] D.J. Wingham, C.R. Francis, S. Baker, C. Bouzinac, D. Brockley, R. Cullen, P. de Chateau-Thierry, S.W. Laxon, U. Mallow, C. Mavrocordatos, L. Phalippou, G. Ratier, L. Rey, F. Rostan, P. Viau, and D.W. Wallis, "CryoSat: A mission to determine the fluctuations in earth's land and marine ice fields," Adv. Space Res., vol. 37, no. 4, pp. 841-871, 2006, Natural Hazards and Oceanographic Processes from Satellite Data.

[4] R.K. Raney, "The delay/Doppler radar altimeter," IEEE Trans. Geoscienc. Remote Sens., vol. 36, no. 5, pp. 15781588, Sep 1998.

[5] R. Penrose, "A generalized inverse for matrices," Proceedings of the Cambridge Philosophical Society, vol. 51, pp. 406-413, 1955.

[6] C. Ray, C. Martin-Puig, M. P. Clarizia, G. Ruffini, S. Dinardo, C. Gommenginger, and J. Benveniste, "SAR Altimeter backscattered waveform model," IEEE Trans. Geoscienc. Remote Sens., vol. 53, no. 2, pp. 911-919, Feb 2015.

[7] F. Boy, J. D. Desjonquéres, N. Picot, T. Moreau, and M. Raynal, "Cryosat-2 sar-mode over oceans: Processing methods, global assessment, and benefits," IEEE Transactions on Geoscience and Remote Sensing, vol. PP, no. 99, pp. 1-11, 2016.

[8] Yongcun Cheng, Jean Tournadre, Xiaofeng Li, Qing Xu, and Bertrand Chapron, "Impacts of oil spills on altimeter waveforms and radar backscatter cross-section," Journal of Geophysical Research: Oceans, pp. n/a-n/a, 2017. 